\title{
FACTORS ASSOCIATED WITH INCREASE IN ALCOHOL CONSUMPTION DURING FIRST MONTHS OF COVID-19 PANDEMIC AMONG ONLINE SOCIAL MEDIA USERS IN RUSSIA
}

\author{
Gil AU $\bowtie$, Demin AK
}

Sechenov First Moscow State Medical University (Sechenov University), Moscow, Russia

\begin{abstract}
The significant proportion of the Russian population are active online social media users. Changes in alcohol consumption in this target group during the COVID-19 pandemic remain understudied. The aim of this survey was to investigate changes in alcohol consumption and factors associated with the increase in alcohol use among online social media users in Russia during the first months of the COVID-19 pandemic. An online survey was conducted among 1,518 users of online social networking services popular in Russia from June to September 2020. The survey revealed that $35.4 \%$ of men and $25.6 \%$ of women started drinking more frequently during the first months of the pandemic; $24.9 \%$ of men and $17.7 \%$ of women increased their usual consumption (volume) of alcohol on a typical drinking occasion, whereas $28.5 \%$ of men and $27.9 \%$ of women increased the frequency of heavy episodic drinking. Adjusted binary logistic regression analysis revealed a positive association between the increase in the frequency of alcohol consumption and the following factors: age from 18 to 29 years (OR: 1.710 ; $95 \%$ Cl: 1.002-2.917), severe restrictions in everyday private life (OR: $3.127 ; 95 \% \mathrm{Cl}: 1.011-9.675)$ and severe negative professional or financial consequences due to the spread of SARS-CoV-2 (OR: 2.247; 95\% Cl: 1.131-4.465). The odds of an increase in the frequency of heavy episodic drinking were more than twice higher (OR: 2.329; 95\% Cl: 1.001-5.428) among those who had experienced severe negative consequences of the pandemic to their professional and financial situation. Higher typical frequency and usual consumption (volume) of alcohol on a typical drinking occasion and higher typical frequency of heavy episodic drinking before the pandemic were positively significantly associated with the increase in these parameters of alcohol consumption during the first months of the pandemic. In times of large-scale epidemics and public health crises, it is advisable to consider the possibility of implementing screening and brief interventions, including via online social media, to prevent problems associated with alcohol use.
\end{abstract}

Keywords: alcohol, COVID-19, coronavirus, pandemic, social media, Facebook, VKontakte, Odnoklassniki, Twitter, Russia

Author contributions: Gil AU planned the study, designed the electronic questionnaire form, collected date, performed statistical analysis, interpreted the results and wrote the manuscript; Demin AK interpreted the results and wrote the manuscript.

Acknowledgements: the authors thank Uri and Raisa Gil for their help in disseminating information about the survey in online social media, for their critical comments on the interpretation and discussion of the results.

$\square$ Correspondence should be addressed: Artyom U. Gil

Trubetskaya, 8, str. 2, Moscow, 119991, Russia; gil.artyom@gmail.com

Received: 21.11.2021 Accepted: 19.12.2021 Published online: 30.12.2021

DOI: $10.24075 /$ brsmu.2021.064

\section{ФАКТОРЫ, ВЗАИМОСВЯЗАННЫЕ С РОСТОМ ПОТРЕБЛЕНИЯ АЛКОГОЛЯ В ПЕРВЫЕ МЕСЯЦЫ ПАНДЕМИИ СОVID-19 СРЕДИ ПОЛЬЗОВАТЕЛЕЙ СОЦИАЛЬНЫХ ОНЛАЙН-СЕТЕЙ В РОССИИ}

А. Ю. Гиль凶, А. К. Дёмин

Первый Московский государственный медицинский университет имени И. М. Сеченова (Сеченовский Университет), Москва, Россия

Значительная часть населения активно пользуется социальными онлайн-сетями, однако изменения потребления алкоголя в этой целевой группе в период пандемии COVID-19 малоизучены. Целью исследования было оценить изменения потребления алкоголя и фракторов, связанных с ростом его потребления, в первые месяцы пандемии COVID-19 среди пользователей социальных онлайн-сетей в России. В период с июня по сентябрь 2020 г. 1518 пользователей наиболее популярных в России социальных онлайн-сетей прошли опрос в отношении изменений потребления алкоголя в первые месяцы пандемии COVID-19. Выявлено, что в первые месяцы пандемии 35,4\% мужчин и 25,6\% женщин увеличили частоту употребления алкоголя; 24,9\% мужчин и 17,7\% женщин увеличили разовый объем потребления алкоголя, и 28,5\% мужчин и 27,9\% женщин увеличили частоту случаев эпизодического употребления алкоголя в больших разовых количествах. На многофакторном уровне возраст 18-29 лет (ОШ = 1,710; 95\% ДИ = 1,002-2,917), очень сильные ограничения в повседневной жизни $(3,127 ; 1,011-9,675)$ и очень сильные негативные последствия в отношении профессиональной или финансовой ситуации в связи с распространением SARS-CoV-2 $(2,247 ; 1,131-4,465)$ были положительно взаимосвязаны с ростом частоты потребления алкоголя. Шансы увеличения частоты эпизодического употребления алкоголя в больших разовых количествах были более чем в два раза выше $(2,329 ; 1,001-5,428)$ среди лиц, испытавших очень сильные негативные последствия в отношении профессиональной или финансовой ситуации. Более высокие привычные частота употребления алкоголя, разовый объем употребляемого алкоголя и частота эпизодического употребления алкоголя в больших разовых количествах до пандемии были положительно статистически значимо связаны с ростом этих параметров потребления алкоголя в первые месяцы пандемии

Ключевые слова: алкоголь, COVID-19, коронавирус, пандемия, социальные сети, Facebook, ВКонтакте, Одноклассники, Тwitter, Россия

Вклад авторов: А. Ю. Гиль - планирование исследования, разработка электронной формы сбора данных; сбор, обработка и статистический анализ данных; интерпретация результатов, написание статьи; А. К. Дёмин - интерпретация результатов исследования, написание статьи.

Благодарности: авторы благодарят Ю. Я. Гиль и Р. Г. Гиль за помощь в распространении информации об опросе в социальных сетях, за ценные критические замечания в отношении интерпретации и обсуждения результатов исследования.

$\triangle$ Для корреспонденции: Артем Юрьевич Гиль

ул. Трубецкая, д. 8, стр. 2, г. Москва, 119991; gil.artyom@gmail.com

Поступила: 21.11.2021 Статья принята к печати: 19.12.2021 Опубликована онлайн: 30.12.2021

DOI: $10.24075 /$ vrgmu.2021.064 
Decisive action taken to counter COVID-19 in Russia and worldwide during the first months of the pandemic slowed the spread of SARS-CoV-2 infection, but it also had a significant impact on many other aspects of life. During the nationwide Russian lockdown, implemented in the second quarter of 2020, the official unemployment rate grew by $2.1 \%$, reaching $6.4 \%$, and the real disposable income of the population fell by $8.4 \%$ in comparison with the same period in 2019 [1]. Income reduction and complete loss of income were reported by $61 \%$ and $13.5 \%$ of the population, respectively; $9.8 \%$ of the Russian population lost their jobs [2]. According to recent studies investigating the impact of COVID-19 restrictions across countries, the rates of depression, anxiety and stress among some population groups, especially among women, young adults and people with chronic diseases, have increased [3, 4]. In times of crisis, some turn to alcohol to cope with psychoemotional stress; this can heighten the risk of adverse short-term and long-term health effects and negative social consequences [5, 6]. Increased substance use following large-scale disasters is often the sign of people adopting a self-medicating strategy to deal with emotional distress [7-9]. Research warns that the COVID-19 pandemic may lead to a medium- and long-term increase in alcohol consumption, especially among men [10]. Early studies into the effects of the pandemic have discovered an association between poor overall mental health and increased alcohol use [11]. According to a study conducted in Canada, stress was the third most common (44\%) cause of drinking during the pandemic. In the USA, psychological distress caused by the pandemic was associated with increased frequency of alcohol use in both men and women [12]. Research demonstrates that while some proportion of the adult population are increasing their alcohol consumption during the pandemic, an equal proportion are cutting down on alcohol, and $50-70 \%$ of the population are not changing their level of alcohol consumption [11, 13]. Because changes in alcohol consumption during the current pandemic may have serious long-term social and economic implications for individuals, groups of people and society as a whole [14, 15], there is a need to monitor these changes and analyze the contributing factors that come forward during a large-scale crisis.

In Russia, online social networking services are actively used by the significant proportion of the population. This opens up a possibility to rapidly assess alcohol consumption among Russian residents and remotely (i.e., via the Internet) implement brief interventions aimed at preventing health problems and other adverse outcomes associated with alcohol use. The aim of this study was to assess changes in alcohol consumption and the factors associated with the increase in alcohol use in the first months of the COVID-19 pandemic among online social media users in Russia in order to explore the possibility of delivering screening and preventive interventions, including those implemented via the Internet, aimed at identifying and preventing alcohol-related problems during large-scale epidemics and public health crises.

\section{METHODS}

An anonymous online survey was conducted from June 18 to September 30, 2020 among users of online social media popular in Russia (Odnoklassniki, VKontakte, Facebook and Twitter). The following inclusion criteria were applied: age $\geq 18$ years, being an Internet user with or without a user account in the online social media mentioned above, informed consent to participate in the anonymous confidential online survey. Non-inclusion criteria: permanent residence of the respondent outside Russia. Exclusion criteria: refusal to participate or have one's personal data processed at any stage of the study, inconsistent contradictory answers to the questions included in the survey. Any participant could refuse to participate at any stage of the study. The link to the survey was posted in the online social media in various groups, on popular pages and in the news feed. The survey was adapted from the pan-European study of alcohol use during the COVID-19 pandemic [10] and modified to assess alcohol consumption behaviors in the 3 months preceding the survey. A few original questions were added to the questionnaire to assess consumption of unrecorded alcohol, such as the homemade alcohol (samogon, homemade wine, braga), alcohol brought from abroad, falsified and counterfeit alcoholic beverages, alcohol-containing liquids not intended for drinking, and other types of unrecorded alcohol. Changes in alcohol use during the first months of the pandemic were assessed from changes in the amount and frequency of alcohol consumption and the frequency of heavy episodic drinking defined as 6 or more drinks or $60 \mathrm{~g}$ of pure ethanol on a single occasion. Statistical analysis included calculation of descriptive statistics and estimation of the proportion of respondents stratified by sociodemographic or other characteristics who had reduced or increased the frequency of drinking or the amount of alcohol consumed. We also assessed associations between the increase in drinking frequency / amount of consumed alcohol during the first months of the pandemic (dependent variables) and the sociodemographic factors, typical frequency of drinking, the typical amount of consumed alcohol, and the typical frequency of heavy episodic drinking in the past 12 months preceding the pandemic, the perceived strength of COVID-19-associated restrictions in public and everyday private life, stress, negative impact of the pandemic on professional and financial situation, and other adverse consequences of the pandemic (independent variables). The presence, direction, strength and statistical significance of the associations were assessed using unadjusted and adjusted binary logistic regression analysis. Odds ratios (OR) and 95\% confidence intervals $(95 \% \mathrm{Cl})$ were calculated as measures of association. Data processing and statistical analysis were conducted in SPSS v.22 (Chicago, IL; USA).

\section{RESULTS}

Of 1,518 respondents, $57.9 \%$ were women and $42.1 \%$ were men. The majority of the respondents $(87.1 \%)$ had had an alcoholic drink at least once in 12 months preceding the study (Table 1). Over half of the respondents (55.9\%) were 30-49 years old and almost two-thirds had attended and/or completed higher education (63.8\%). More men than women lived in larger settlements $(p=0.010)$ and higher-income households $(p<0.001)$. During the first months of the pandemic, their income had changed or fallen less often than that of women $(p=0.014)$. The usual frequency of drinking, the number of standard drinks consumed on a typical occasion, the frequency of heavy episodic drinking, and the proportion of persons consuming unrecorded alcohol were higher among men than among women $(p<0.001)$. Overall, the male respondents reported they had encountered restrictions in public $(p=0.007)$ and everyday private $(p=0.003)$ life less often than women. However, almost a quarter of men (23.2\%) and a third of women (30.8\%) said they had encountered severe restrictions in public life, whereas one-fifth of men (19.9\%) and a quarter of women (25.4\%) reported having faced severe restrictions in their everyday private life during the first months of the pandemic. Men had experienced the negative effects of the pandemic on their professional or financial situation significantly less frequently than women $(p=0.005)$. Only $30.4 \%$ of men and $23.5 \%$ of women reported they had experienced no negative effects on their professional or financial situation. The pandemic 
was a source of stress for $50.4 \%$ of men and $69.5 \%$ of women $(p<0.001)$. A history of confirmed SARS-CoV-2 infection in a respondent, their family or close friends was reported by $21.9 \%$ of the participants, with no significant differences by sex (Table 1). In the first months of the pandemic, 35.4\% of men and $25.6 \%$ of women had used alcohol more frequently than before the pandemic; $24.9 \%$ of men and $17.7 \%$ of women had increased usual consumption (volume) of alcohol on a typical drinking occasion; the frequency of heavy episodic drinking had increased in $28.5 \%$ of men and $27.9 \%$ of women.

Results of unadjusted and adjusted logistic regression analysis are shown in Tables 2-4.

\section{Factors associated with the increase in the frequency of alcohol consumption during the first months of the pandemic}

After adjustment for confounders, positive statistically significant associations between the increase in the frequency of alcohol consumption during the first months of the pandemic and the following factors were identified: age of 18-29 years (OR: 1.710;
95\% Cl: 1.002-2.917), higher typical frequency of alcohol use before the pandemic (from OR: 3.190, 95\% Cl: 1.887-5.392 for consumption 2-4 times a months, to OR: $18.727,95 \% \mathrm{Cl}$ : 9.639-36.383 for consumption more than 2-3 times a month), higher usual consumption (volume) of alcohol on a typical drinking occasion before the pandemic (from OR: 1.941, 95\% Cl: 1.244-3.029 to OR: 2.234, 95\%Cl: 1.180-4.233), heavy episodic drinking with a frequency of once a month (OR: 2/061; 95\% Cl: 1.157-3.671) and once a week before the pandemic (OR: 2.012; 95\% Cl: 1.081-3.746), severe restrictions in everyday private life due to SARS-CoV-2 containment measures (OR: 3.127; 95\% Cl: 1.011-9.675) and severe negative consequences of the pandemic to the professional or financial situation (OR: 2.247; 95\% Cl: 1.131-4.465, Table 2).

\section{Factors associated with the increase of the usual consumption (volume) of alcohol on a typical drinking occasion during the first months of the pandemic}

Positive statistically significant associations were identified between the increase of the usual consumption (volume) of

Table 1. Sociodemographic characteristics of the respondents and characteristics related to typical alcohol use and the SARS-CoV-2 pandemic, by sex (\%)

\begin{tabular}{|c|c|c|c|c|}
\hline \multirow{2}{*}{ Characteristic } & Men & Women & Both sexes & \multirow{2}{*}{$p^{*}$} \\
\hline & $n(\%)$ & $n(\%)$ & $n(\%)$ & \\
\hline \multicolumn{5}{|c|}{ Age (years) } \\
\hline $18-29$ & $150(23.5)$ & $172(19.6)$ & $322(21.2)$ & \multirow{3}{*}{0.159} \\
\hline $30-49$ & $351(54.9)$ & $497(56.5)$ & $848(55.9)$ & \\
\hline$\geq 50$ & $138(21.6)$ & $210(23.9)$ & $348(22.9)$ & \\
\hline \multicolumn{5}{|c|}{ Education } \\
\hline Secondary or below & $143(22.4)$ & $161(18.3)$ & $304(20.0)$ & \multirow{3}{*}{0.108} \\
\hline Primary /vocational school or college & 95 (14.9) & $151(17.2)$ & $246(16.2)$ & \\
\hline Higher (complete or incomplete) & $401(62.8)$ & $567(64.5)$ & $968(63.8)$ & \\
\hline \multicolumn{5}{|c|}{ Settlement size } \\
\hline$\leq 50,000$ population & $111(17.4)$ & $196(22.3)$ & $307(20.2)$ & \multirow{3}{*}{0.010} \\
\hline 50,000 to 1 million & $251(39.3)$ & $362(41.2)$ & $613(40.4)$ & \\
\hline Over 1 million & $277(43.3)$ & $321(36.5)$ & $598(39.4)$ & \\
\hline \multicolumn{5}{|c|}{ Income per household member (rubles) } \\
\hline$\leq 9,999$ & $130(20.3)$ & $221(25.1)$ & $351(23.1)$ & \multirow{5}{*}{$<0.001$} \\
\hline $10,000-19,999$ & $157(24.6)$ & $225(25.6)$ & $382(25.2)$ & \\
\hline $20,000-39,999$ & $136(21.3)$ & $226(25.7)$ & $362(23.8)$ & \\
\hline $40,000-59,999$ & $116(18.2)$ & $128(14.6)$ & $244(16.1)$ & \\
\hline$\geq 60,000$ & $100(15.6)$ & $79(9.0)$ & 179 (11.8) & \\
\hline \multicolumn{5}{|c|}{ Changes in the average monthly household income since the beginning of the SARS-CoV-2 pandemic } \\
\hline Did not change or increased & $341(53.4)$ & $402(45.7)$ & $743(48.9)$ & \multirow{4}{*}{0.014} \\
\hline Slightly fell & $124(19.4)$ & $187(21.3)$ & $311(20.5)$ & \\
\hline Moderately fell & $79(12.4)$ & $149(17.0)$ & $228(15.0)$ & \\
\hline Dropped significantly & $95(14.9)$ & $141(16.0)$ & $236(15.5)$ & \\
\hline \multicolumn{5}{|c|}{ Typical frequency of alcohol use in the past 12 months before pandemic } \\
\hline Never & $84(13.4)$ & $108(12.6)$ & $192(12.9)$ & \multirow{5}{*}{$<0.001$} \\
\hline Once a month or less frequently & $121(19.3)$ & $314(36.5)$ & $435(29.3)$ & \\
\hline 2-4 times a month & $183(29.2)$ & $227(26.4)$ & $410(27.6)$ & \\
\hline 2-3 times a week & $118(18.8)$ & $116(13.5)$ & $234(15.7)$ & \\
\hline More often than 2-3 times a week & $121(19.3)$ & $95(11.0)$ & $216(14.5)$ & \\
\hline \multicolumn{5}{|c|}{ Number of standard alcoholic drinks consumed on a typical drinking occasion in the past 12 months before pandemic ${ }^{\star \star}$} \\
\hline $1-2$ & $136(26.0)$ & $424(57.1)$ & $560(44.2)$ & \multirow{3}{*}{$<0.001$} \\
\hline $3-6$ & $256(48.9)$ & 256 (34.5) & $512(40.4)$ & \\
\hline $7+$ & $131(25.0)$ & $63(8.5)$ & 194 (15.3) & \\
\hline
\end{tabular}


Table 1 cont.

\begin{tabular}{|c|c|c|c|c|}
\hline \multirow{2}{*}{ Characteristic } & Men & Women & Both sexes & \multirow{2}{*}{$p^{*}$} \\
\hline & $n(\%)$ & $n(\%)$ & $n(\%)$ & \\
\hline \multicolumn{5}{|c|}{ Typical frequency of heavy episodic drinking (6 or more standard drinks on a single occasion) in the past 12 months before pandemic } \\
\hline Never & $191(31.3)$ & $446(52.3)$ & $637(43.6)$ & \multirow{5}{*}{$<0.001$} \\
\hline Less than once a month & $124(20.3)$ & $176(20.7)$ & $300(20.5)$ & \\
\hline Once a month & $96(15.7)$ & $102(12.0)$ & $198(13.5)$ & \\
\hline Once a week & $136(22.3)$ & 96 (11.3) & $232(15.9)$ & \\
\hline Every day or almost every day & $63(10.3)$ & $32(3.8)$ & $95(6.5)$ & \\
\hline \multicolumn{5}{|l|}{ Reported consumption of unrecorded alcohol } \\
\hline Yes & $320(51.3)$ & 359 (41.9) & $679(45.8)$ & \multirow{2}{*}{$<0.001$} \\
\hline No & $304(48.7)$ & $498(58.1)$ & $802(54.2)$ & \\
\hline \multicolumn{5}{|c|}{ Reported encountering COVID-19-related restrictions in public life in the past 3 months } \\
\hline No restrictions & $61(9.7)$ & $65(7.5)$ & $126(8.4)$ & \multirow{4}{*}{0.007} \\
\hline Slight restrictions & $222(35.3)$ & $267(30.8)$ & $489(32.7)$ & \\
\hline Moderate restrictions & $200(31.8)$ & $267(30.8)$ & $467(31.2)$ & \\
\hline Severe restrictions & $146(23.2)$ & $267(30.8)$ & $413(27.6)$ & \\
\hline \multicolumn{5}{|c|}{ Reported encountering COVID-19-related restrictions in their everyday private life in the past 3 months } \\
\hline No restrictions & $81(12.8)$ & $72(8.3)$ & $153(10.2)$ & \multirow{4}{*}{0.003} \\
\hline Slight restrictions & $255(40.3)$ & $317(36.6)$ & $572(38.2)$ & \\
\hline Moderate restrictions & $171(27.0)$ & $257(29.7)$ & $428(28.6)$ & \\
\hline Severe restrictions & $126(19.9)$ & $220(25.4)$ & $346(23.1)$ & \\
\hline \multicolumn{5}{|c|}{ Reported negative consequences of the pandemic to their professional or financial situation in the past 3 months } \\
\hline No negative consequences & $189(30.4)$ & $203(23.5)$ & $392(26.4)$ & \multirow{4}{*}{0.005} \\
\hline Slight negative consequences & $235(37.8)$ & $336(38.9)$ & $571(38.5)$ & \\
\hline Moderate negative consequences & $114(18.4)$ & $163(18.9)$ & $277(18.7)$ & \\
\hline Severe negative consequences & $83(13.4)$ & $161(18.7)$ & $244(16.4)$ & \\
\hline \multicolumn{5}{|c|}{ Reported confirmed SARS-CoV-2 infection in themselves, their family or close friends in the past 3 months } \\
\hline Yes & $137(22.1)$ & $186(21.8)$ & $323(21.9)$ & \multirow{2}{*}{0.949} \\
\hline No & $484(77.9)$ & 667 (78.2) & $1151(78.1)$ & \\
\hline \multicolumn{5}{|c|}{ Reported stress due to the spread of SARS-CoV-2 in the past 3 months } \\
\hline Yes & $292(50.4)$ & $574(69.5)$ & $866(61.6)$ & \multirow{2}{*}{$<0.001$} \\
\hline No & $287(49.6)$ & $252(30.5)$ & $539(38.4)$ & \\
\hline
\end{tabular}

Note: ${ }^{*}$ - significance of differences between the groups was assessed using $x^{2}$-test for heterogeneity; ${ }^{\star}$ — among alcohol drinkers (those who consumed alcohol at least once in the past 12 months).

Table 2. Associations of sociodemographic factors, typical frequency and volume of alcohol use, unrecorded alcohol consumption, negative consequences of the COVID-19 pandemic with the increase in the frequency of alcohol consumption in the first months of the COVID-19 pandemic, 2020, (OR, 95\% Cl)

\begin{tabular}{|c|c|c|c|c|}
\hline \multirow[t]{2}{*}{ Variables } & \multicolumn{2}{|c|}{$\begin{array}{l}\text { Respondents who increased } \\
\text { frequency of alcohol } \\
\text { consumption }\end{array}$} & \multirow{2}{*}{$\begin{array}{l}\text { Unadjusted } \\
\text { OR }(95 \% \mathrm{Cl})\end{array}$} & \multirow{2}{*}{$\begin{array}{c}\text { Adjusted } \\
\text { OR }(95 \% \mathrm{Cl})\end{array}$} \\
\hline & $n / \mathrm{N}$ & $\%$ & & \\
\hline \multicolumn{5}{|c|}{ Sex } \\
\hline Male & $191 / 539$ & 35.4 & $1.511(1.188-1.921)$ & $0.946(0.664-1.348)$ \\
\hline Female & $198 / 743$ & 26.6 & 1.0 & 1.0 \\
\hline \multicolumn{5}{|c|}{ Age (years) } \\
\hline $18-29$ & $91 / 267$ & 34.1 & $1.559(1.076-2.257)$ & $1.710(1.002-2.917)$ \\
\hline $30-49$ & $228 / 734$ & 31.1 & $1.358(0.994-1.856)$ & $1.082(0.690-1.698)$ \\
\hline$\geq 50$ & $70 / 281$ & 24.9 & 1.0 & 1.0 \\
\hline \multicolumn{5}{|c|}{ Education } \\
\hline Secondary or below & $69 / 236$ & 29.2 & $1.610(1.027-2.525)$ & $1.514(0.807-2.839)$ \\
\hline Primary /vocational school or college & $39 / 191$ & 20.4 & 1.0 & 1.0 \\
\hline Higher (complete or incomplete) & $281 / 855$ & 32.9 & 1.908 (1.305-2.789) & $1.644(0.952-2.842)$ \\
\hline \multicolumn{5}{|c|}{ Settlement size } \\
\hline$\leq 50,000$ population & $54 / 240$ & 22.5 & $0.506(0.356-0.718)$ & $0.952(0.576-1.574)$ \\
\hline
\end{tabular}




\begin{tabular}{|c|c|c|c|c|}
\hline \multirow[t]{2}{*}{ Variables } & \multicolumn{2}{|c|}{$\begin{array}{l}\text { Respondents who increased } \\
\text { frequency of alcohol } \\
\text { consumption }\end{array}$} & \multirow{2}{*}{$\begin{array}{l}\text { Unadjusted } \\
\text { OR }(95 \% \text { Cl) }\end{array}$} & \multirow{2}{*}{$\begin{array}{c}\text { Adjusted } \\
\text { OR }(95 \% \mathrm{Cl})\end{array}$} \\
\hline & $n / N$ & $\%$ & & \\
\hline 50,000 to 1 million & $141 / 510$ & 27.6 & $0.666(0.512-0.865)$ & $1.272(0.871-1.858)$ \\
\hline Over 1 million & $194 / 532$ & 36.5 & 1.0 & 1.0 \\
\hline \multicolumn{5}{|c|}{ Income per household member (rubles) } \\
\hline$\leq 9,999$ & $73 / 276$ & 26.4 & $0.622(0.409-0.944)$ & $1.048(0.561-1.957)$ \\
\hline $10,000-19,999$ & $83 / 305$ & 27.2 & $0.646(0.430-0.972)$ & $1.009(0.569-1.791)$ \\
\hline $20,000-39,999$ & $101 / 321$ & 31.5 & $0.794(0.533-1.181)$ & $1.222(0.705-2.117)$ \\
\hline $40,000-59,999$ & $73 / 219$ & 33.3 & $0.864(0.564-0.324)$ & $0.934(0.528-1.650)$ \\
\hline$\geq 60,000$ & $59 / 161$ & 36.6 & 1.0 & 1.0 \\
\hline \multicolumn{5}{|c|}{ Changes in the average monthly household income since the beginning of the SARS-CoV-2 pandemic } \\
\hline Did not change or increased & $166 / 628$ & 26.4 & 1.0 & 1.0 \\
\hline Slightly fell & $84 / 258$ & 32.6 & $1.344(0.980-1.841)$ & $1.074(0.687-1.680)$ \\
\hline Moderately fell & $58 / 194$ & 29.9 & $1.187(0.832-1.693)$ & $0.822(0.485-1.393)$ \\
\hline Dropped significantly & $81 / 202$ & 40.1 & $1.863(1.336-2.598)$ & $1.432(0.803-2.552)$ \\
\hline \multicolumn{5}{|c|}{ Typical frequency of alcohol use in the past 12 months before pandemic } \\
\hline Once a month or less frequently & $32 / 430$ & 7.4 & 1.0 & 1.0 \\
\hline $2-4$ times a month & $110 / 404$ & 27.2 & $4.653(3.053-7.093)$ & $3.190(1.887-5.392)$ \\
\hline $2-3$ times a week & $115 / 233$ & 49.4 & $12.121(7.788-18.865)$ & $7.017(3.898-12.632)$ \\
\hline More often than 2-3 times a week & $132 / 215$ & 61.4 & $19.780(12.576-31.110)$ & $18.727(9.639-36.383)$ \\
\hline \multicolumn{5}{|c|}{ Number of standard alcoholic drinks consumed on a typical drinking occasion in the past 12 months before pandemic } \\
\hline $1-2$ & $84 / 517$ & 16.2 & 1.0 & 1.0 \\
\hline $3-6$ & $198 / 505$ & 39.2 & $3.325(2.477-4.461)$ & $1.941(1.244-3.029)$ \\
\hline $7+$ & $91 / 186$ & 48.9 & $4.938(3.409-7.152)$ & $2.234(1.180-4.233)$ \\
\hline \multicolumn{5}{|c|}{ Typical frequency of heavy episodic drinking (6 or more standard drinks on a single occasion) in the past 12 months before pandemic } \\
\hline Never & $61 / 441$ & 13.8 & 1.0 & 1.0 \\
\hline Less than once a month & $69 / 289$ & 23.9 & $1.954(1.333-2.864)$ & $1.184(0.712-1.969)$ \\
\hline Once a month & $77 / 192$ & 40.1 & $4.171(2.809-6.194)$ & $2.061(1.157-3.671)$ \\
\hline Once a week & $119 / 229$ & 52.0 & $6.739(4.634-9.801)$ & $2.012(1.081-3.746)$ \\
\hline Every day or almost every day & $51 / 92$ & 55.4 & $7.749(4.738-12.674)$ & $1.094(0.444-2.695)$ \\
\hline \multicolumn{5}{|c|}{ Reported consumption of unrecorded alcohol } \\
\hline Yes & $221 / 642$ & 34.4 & $1.498(1.174-1.912)$ & $1.246(0.897-1.730)$ \\
\hline No & $158 / 609$ & 25.9 & 1.0 & 1.0 \\
\hline \multicolumn{5}{|c|}{ Reported encountering COVID-19-related restrictions in public life in the past 3 months } \\
\hline No restrictions & $17 / 87$ & 19.5 & 1.0 & 1.0 \\
\hline Slight restrictions & $84 / 407$ & 20.6 & $1.071(0.598-1.916)$ & $0.703(0.250-1.977)$ \\
\hline Moderate restrictions & $133 / 413$ & 32.2 & $1.956(1.108-3.454)$ & $1.068(0.349-3.270)$ \\
\hline Severe restrictions & $151 / 363$ & 41.6 & $2.933(1.659-5.184)$ & $1.020(0.315-3.299)$ \\
\hline \multicolumn{5}{|c|}{ Reported encountering COVID-19-related restrictions in their everyday private life in the past 3 months } \\
\hline No restrictions & $18 / 111$ & 16.2 & 1.0 & 1.0 \\
\hline Slight restrictions & $101 / 470$ & 21.5 & $1.414(0.815-2.453)$ & $1.636(0.622-4.304)$ \\
\hline Moderate restrictions & $133 / 385$ & 34.5 & $2.727(1.579-4.711)$ & $2.594(0.890-7.564)$ \\
\hline Severe restrictions & $134 / 304$ & 44.1 & $4.073(2.342-7.081)$ & $3.127(1.011-9.675)$ \\
\hline \multicolumn{5}{|c|}{ Reported negative consequences of the pandemic to their professional or financial situation in the past 3 months } \\
\hline No negative consequences & $65 / 318$ & 20.4 & 1.0 & 1.0 \\
\hline Slight negative consequences & $142 / 492$ & 28.9 & $1.579(1.129-2.208)$ & $1.240(0.772-1.990)$ \\
\hline Moderate negative consequences & $87 / 239$ & 36.4 & $2.228(1.525-3.255)$ & $1.524(0.848-2.739)$ \\
\hline Severe negative consequences & $89 / 211$ & 42.2 & $2.839(1.930-4.177)$ & $2.247(1.131-4.465)$ \\
\hline \multicolumn{5}{|c|}{ Reported confirmed SARS-CoV-2 infection in themselves, their family or close friends in the past 3 months } \\
\hline Yes & $82 / 278$ & 29.5 & $0.956(0.714-1.280)$ & $0.846(0.575-1.245)$ \\
\hline No & 295/969 & 30.4 & 1.0 & 1.0 \\
\hline \multicolumn{5}{|c|}{ Reported stress due to the spread of SARS-CoV-2 } \\
\hline Yes & $254 / 746$ & 34.0 & $1.587(1.218-2.068)$ & $1.306(0.892-1.911)$ \\
\hline No & $108 / 440$ & 24.5 & 1.0 & 1.0 \\
\hline
\end{tabular}


alcohol on a typical drinking occasion during the pandemic and the following factors: drinking alcohol 2 times a week and more often before the pandemic (from OR: 2.587; 95\% Cl: $1.360-4.918$ to OR: $12.021 ; 95 \% \mathrm{Cl}: 5.712-25.300)$, consuming 3 or more alcoholic drinks on a typical drinking occasion before the pandemic (for 3-6 drinks OR: 2.145; 95\% Cl: 1.270-3.623; for 7 or more drinks OR: 2.922; 95\% Cl: 1.448-5.894) and increased frequency (once a week) of heavy episodic drinking (OR: 2.380; 95\% Cl: 1.180-4.800) (Table 3).

Table 3. Associations of sociodemographic factors, typical frequency and volume of alcohol use, consumption of unrecorded alcohol, negative consequences of the COVID-19 pandemic with the increase in the usual consumption (volume) of alcohol on a typical drinking occasion in the first months of the COVID-19 pandemic, 2020, (OR, 95\% Cl)

\begin{tabular}{|c|c|c|c|c|}
\hline \multirow[t]{2}{*}{ Variables } & \multicolumn{2}{|c|}{$\begin{array}{l}\text { Respondents who increased } \\
\text { the usual consumption } \\
\text { (volume) of alcohol on a } \\
\text { typical drinking occasion }\end{array}$} & \multirow{2}{*}{$\begin{array}{l}\text { Unadjusted } \\
\text { OR }(95 \% \mathrm{Cl})\end{array}$} & \multirow{2}{*}{$\begin{array}{c}\text { Adjusted } \\
\text { OR }(95 \% \mathrm{Cl})\end{array}$} \\
\hline & $n / \mathrm{N}$ & $\%$ & & \\
\hline \multicolumn{5}{|c|}{ Sex } \\
\hline Male & $129 / 519$ & 24.9 & $1.537(1.166-2.026)$ & $0.875(0.592-1.295)$ \\
\hline Female & $127 / 717$ & 17.7 & 1.0 & 1.0 \\
\hline \multicolumn{5}{|c|}{ Age (years) } \\
\hline $18-29$ & $55 / 261$ & 21.1 & $1.353(0.872-2.100)$ & $1.609(0.875-2.961)$ \\
\hline $30-49$ & $157 / 708$ & 22.2 & $1.444(0.999-2.088)$ & $1.396(0.838-2.325)$ \\
\hline$\geq 50$ & $44 / 267$ & 16.5 & 1.0 & 1.0 \\
\hline \multicolumn{5}{|c|}{ Education } \\
\hline Secondary or below & $49 / 244$ & 21.9 & $1.680(0.990-2.851)$ & $1.810(0.884-3.703)$ \\
\hline Primary /vocational school or college & $25 / 175$ & 14.3 & 1.0 & 1.0 \\
\hline Higher (complete or incomplete) & $182 / 837$ & 21.7 & $1.667(1.059-2.625)$ & $1.797(0.950-3.398)$ \\
\hline \multicolumn{5}{|c|}{ Settlement size } \\
\hline$\leq 50,000$ population & $39 / 232$ & 16.8 & $0.614(0.412-0.915)$ & $0.986(0.568-1.709)$ \\
\hline 50,000 to 1 million & $90 / 491$ & 18.3 & $0.682(0.503-0.925)$ & $1.017(0.664-1.558)$ \\
\hline Over 1 million & $127 / 513$ & 24.8 & 1.0 & 1.0 \\
\hline \multicolumn{5}{|c|}{ Income per household member (rubles) } \\
\hline$\leq 9,999$ & $54 / 259$ & 20.8 & $0.926(0.573-1.497)$ & $1.300(0.647-2.609)$ \\
\hline $10,000-19,999$ & $60 / 295$ & 20.3 & $0.897(0.561-1.436)$ & $1.434(0.755-2.723)$ \\
\hline $20,000-39,999$ & $66 / 310$ & 21.3 & $0.951(0.598-1.511)$ & $1.473(0.794-2.734)$ \\
\hline $40,000-59,999$ & $41 / 214$ & 19.2 & $0.833(0.502-1.383)$ & $0.887(0.460-1.710)$ \\
\hline$\geq 60,000$ & $35 / 158$ & 22.2 & 1.0 & 1.0 \\
\hline \multicolumn{5}{|c|}{ Changes in the average monthly household income since the beginning of the SARS-CoV-2 pandemic } \\
\hline Did not change or increased & $102 / 603$ & 16.9 & 1.0 & 1.0 \\
\hline Slightly fell & $50 / 255$ & 19.6 & $0.417(0.288-0.603)$ & $0.917(0.550-1.528)$ \\
\hline Moderately fell & $41 / 186$ & 22.0 & $0.499(0.324-0.769)$ & $0.990(0.559-1.753)$ \\
\hline Dropped significantly & $63 / 192$ & 32.8 & $0.579(0.366-0.916)$ & $1.686(0.915-3.104)$ \\
\hline \multicolumn{5}{|c|}{ Typical frequency of alcohol use in the past 12 months before pandemic } \\
\hline Once a month or less frequently & $21 / 421$ & 5.0 & 1.0 & 1.0 \\
\hline $2-4$ times a month & $66 / 386$ & 17.1 & $3.929(2.353-6.559)$ & $2.587(1.360-4.918)$ \\
\hline 2-3 times a week & $70 / 225$ & 31.1 & $8.602(5.105-14.494)$ & $4.617(2.309-9.233)$ \\
\hline More often than 2-3 times a week & $99 / 204$ & 48.5 & $17.959(10.703-30.136)$ & $12.021(5.712-25.300)$ \\
\hline \multicolumn{5}{|c|}{ Number of standard alcoholic drinks consumed on a typical drinking occasion in the past 12 months before pandemic } \\
\hline $1-2$ & $44 / 509$ & 8.6 & 1.0 & 1.0 \\
\hline $3-6$ & $132 / 492$ & 26.8 & $3.875(2.682-5.598)$ & $2.145(1.270-3.623)$ \\
\hline $7+$ & $76 / 186$ & 40.9 & $7.302(4.771-11.174)$ & $2.922(1.448-5.894)$ \\
\hline \multicolumn{5}{|c|}{ Typical frequency of heavy episodic drinking ( 6 or more drinks on a single occasion) in the past 12 months before pandemic } \\
\hline Never & $32 / 440$ & 7.3 & 1.0 & 1.0 \\
\hline Less than once a month & $45 / 284$ & 15.8 & $2.401(1.485-3.882)$ & $1.434(0.781-2.632)$ \\
\hline Once a month & 43/192 & 22.4 & $3.680(2.244-6.034)$ & $1.876(0.956-3.681)$ \\
\hline Once a week & $90 / 228$ & 39.5 & $8.315(5.317-13.004)$ & $2.380(1.180-4.800)$ \\
\hline Every day or almost every day & $46 / 92$ & 50.0 & $12.750(7.397-21.978)$ & $2.376(0.905-6.240)$ \\
\hline \multicolumn{5}{|c|}{ Reported consumption of unrecorded alcohol } \\
\hline Yes & $145 / 620$ & 23.4 & $1.385(1.046-1.834)$ & $0.906(0.628-1.308)$ \\
\hline No & $106 / 587$ & 18.1 & 1.0 & 1.0 \\
\hline
\end{tabular}




\begin{tabular}{|c|c|c|c|c|}
\hline \multirow[t]{2}{*}{ Variables } & \multicolumn{2}{|c|}{$\begin{array}{l}\text { Respondents who increased } \\
\text { the usual consumption } \\
\text { (volume) of alcohol on a } \\
\text { typical drinking occasion }\end{array}$} & \multirow{2}{*}{$\begin{array}{l}\text { Unadjusted } \\
\text { OR }(95 \% \mathrm{Cl})\end{array}$} & \multirow{2}{*}{$\begin{array}{c}\text { Adjusted } \\
\text { OR }(95 \% \mathrm{Cl})\end{array}$} \\
\hline & $n / N$ & $\%$ & & \\
\hline \multicolumn{5}{|c|}{ Reported encountering COVID-19-related restrictions in public life in the past 3 months } \\
\hline No restrictions & $13 / 84$ & 15.5 & 1.0 & 1.0 \\
\hline Slight restrictions & $49 / 378$ & 13.0 & $0.813(0.419-1.579)$ & $1.092(0.341-3.497)$ \\
\hline Moderate restrictions & $88 / 408$ & 21.6 & $1.502(0.795-2.839)$ & $1.472(0.409-5.298)$ \\
\hline Severe restrictions & $104 / 357$ & 29.1 & $2.245(1.191-4.231)$ & $1.539(0.407-5.820)$ \\
\hline \multicolumn{5}{|c|}{ Reported encountering COVID-19-related restrictions in their everyday private life in the past 3 months } \\
\hline No restrictions & $16 / 108$ & 14.8 & 1.0 & 1.0 \\
\hline Slight restrictions & $55 / 449$ & 12.2 & $0.803(0.440-1.464)$ & $0.809(0.282-2.318)$ \\
\hline Moderate restrictions & $93 / 374$ & 24.9 & $1.903(1.065-3.400)$ & $1.767(0.550-5.681)$ \\
\hline Severe restrictions & $91 / 296$ & 30.7 & $2.552(1.421-4.584)$ & $1.737(0.509-5.931)$ \\
\hline \multicolumn{5}{|c|}{ Reported negative consequences of the pandemic to their professional or financial situation in the past 3 months } \\
\hline No negative consequences & $42 / 308$ & 13.6 & 1.0 & 1.0 \\
\hline Slight negative consequences & $83 / 472$ & 17.6 & $1.351(0.903-2.021)$ & $0.976(0.566-1.683)$ \\
\hline Moderate negative consequences & $60 / 232$ & 25.9 & $2.209(1.425-3.425)$ & $1.453(0.761-2.776)$ \\
\hline Severe negative consequences & $67 / 205$ & 32.7 & $3.075(1.986-4.761)$ & $2.072(0.983-4.369)$ \\
\hline \multicolumn{5}{|c|}{ Reported confirmed SARS-CoV-2 infection in themselves, their family or close friends in the past 3 months } \\
\hline Yes & $47 / 270$ & 17.4 & $0.772(0.544-1.098)$ & $0.686(0.442-1.064)$ \\
\hline No & $200 / 933$ & 21.4 & 1.0 & 1.0 \\
\hline \multicolumn{5}{|c|}{ Reported stress due to the spread of SARS-CoV-2 } \\
\hline Yes & $168 / 726$ & 23.1 & $1.523(1.116-2.077)$ & $1.265(0.819-1.952)$ \\
\hline No & $69 / 418$ & 16.5 & 1.0 & 1.0 \\
\hline
\end{tabular}

Table 4. Associations of sociodemographic factors, typical frequency and volume of alcohol use, consumption of unrecorded alcohol, negative consequences of the COVID-19 pandemic with the increase in the frequency of heavy episodic drinking in the first months of the COVID-19 pandemic, 2020, (OR, 95\% Cl)*

\begin{tabular}{|c|c|c|c|c|}
\hline \multirow[t]{2}{*}{ Variable } & \multicolumn{2}{|c|}{$\begin{array}{l}\text { Respondents who increased } \\
\text { the frequency of heavy } \\
\text { episodic drinking }\end{array}$} & \multirow{2}{*}{$\begin{array}{l}\text { Unadjusted } \\
\text { OR }(95 \% \mathrm{Cl})\end{array}$} & \multirow{2}{*}{$\begin{array}{c}\text { Adjusted } \\
\text { OR }(95 \% \mathrm{Cl})\end{array}$} \\
\hline & $n / \mathrm{N}$ & $\begin{array}{c}\text { OR }(95 \% \\
\text { Cl) }\end{array}$ & & \\
\hline \multicolumn{5}{|c|}{ Sex } \\
\hline Male & $117 / 411$ & 28.5 & $1.028(0.755-1.400)$ & $0.759(0.497-1.159)$ \\
\hline Female & $108 / 387$ & 27.9 & 1.0 & 1.0 \\
\hline \multicolumn{5}{|c|}{ Age (years) } \\
\hline $18-29$ & $50 / 173$ & 28.9 & $1.370(0.831-2.260)$ & $1.732(0.867-3.462)$ \\
\hline $30-49$ & $140 / 472$ & 29.7 & $1.422(0.929-2.176)$ & $1.363(0.762-2.437)$ \\
\hline$\geq 50$ & $35 / 153$ & 22.9 & 1.0 & 1.0 \\
\hline \multicolumn{5}{|c|}{ Education } \\
\hline Secondary or below & $47 / 171$ & 27.5 & $1.401(0.792-2.477)$ & $1.487(0.700-3.157)$ \\
\hline Primary /vocational school or college & $23 / 108$ & 21.3 & 1.0 & 1.0 \\
\hline Higher (complete or incomplete) & $155 / 519$ & 29.9 & $1.574(0.957-2.588)$ & $1.476(0.753-2.894)$ \\
\hline \multicolumn{5}{|c|}{ Settlement size } \\
\hline$\leq 50,000$ population & $36 / 153$ & 23.5 & $0.698(0.451-1.080)$ & $1.043(0.567-1.918)$ \\
\hline 50,000 to 1 million & $81 / 292$ & 27.7 & $0.871(0.619-1.226)$ & $1.534(0.944-2.493)$ \\
\hline Over 1 million & $108 / 353$ & 30.6 & 1.0 & 1.0 \\
\hline \multicolumn{5}{|c|}{ Income per household member (rubles) } \\
\hline$\leq 9,999$ & $53 / 177$ & 29.9 & $1.187(0.688-2.047)$ & $1.458(0.680-3.130)$ \\
\hline $10,000-19,999$ & $48 / 184$ & 26.1 & $0.980(0.566-1.698)$ & $1.210(0.589-2.488)$ \\
\hline 20,000-39,999 & $57 / 195$ & 29.2 & $1.147(0.670-1.964)$ & $1.299(0.650-2.596)$ \\
\hline $40,000-59,999$ & $40 / 140$ & 28.6 & $1.111(0.627-1.970)$ & $1.218(0.593-2.501)$ \\
\hline$\geq 60,000$ & $27 / 102$ & 26.5 & 1.0 & 1.0 \\
\hline
\end{tabular}




\begin{tabular}{|c|c|c|c|c|}
\hline \multicolumn{5}{|c|}{ Changes in the average monthly household income since the beginning of the SARS-CoV-2 pandemic } \\
\hline Did not change or increased & $90 / 380$ & 23.7 & 1.0 & 1.0 \\
\hline Slightly fell & $43 / 167$ & 25.7 & $1.117(0.734-1.700)$ & $1.087(0.618-1.914)$ \\
\hline Moderately fell & $41 / 130$ & 31.5 & $1.484(0.957-2.303)$ & $1.246(0.664-2.337)$ \\
\hline Dropped significantly & $51 / 121$ & 42.1 & $2.348(1.525-3.614)$ & $1.624(0.812-3.246)$ \\
\hline \multicolumn{5}{|c|}{ Typical frequency of alcohol use in the past 12 months before pandemic } \\
\hline Once a month or less frequently & $13 / 142$ & 9.2 & 1.0 & 1.0 \\
\hline $2-4$ times a month & $56 / 277$ & 20.2 & $2.514(1.324-4.775)$ & $1.315(0.566-3.056)$ \\
\hline $2-3$ times a week & $64 / 192$ & 33.3 & $4.962(2.605-9.451)$ & $2.756(1.137-6.679)$ \\
\hline More often than 2-3 times a week & $92 / 187$ & 49.2 & $9.610(5.076-18.194)$ & $6.581(2.585-16.749)$ \\
\hline \multicolumn{5}{|c|}{ Number of standard alcoholic drinks consumed on a typical drinking occasion in the past 12 months before pandemic } \\
\hline $1-2$ & $21 / 171$ & 12.3 & 1.0 & 1.0 \\
\hline $3-6$ & $122 / 403$ & 30.3 & $3.101(1.874-5.132)$ & $1.583(0.836-2.999)$ \\
\hline $7+$ & $78 / 184$ & 42.4 & $5.256(3.056-9.040)$ & $2.202(1.014-4.779)$ \\
\hline \multicolumn{5}{|c|}{ Typical frequency of heavy episodic drinking (6 or more drinks on a single occasion) in the past 12 months before pandemic } \\
\hline Never & $38 / 288$ & 13.2 & 1.0 & 1.0 \\
\hline Less than once a month & $46 / 190$ & 24.2 & $2.102(1.306-3.383)$ & $2.561(1.341-4.893)$ \\
\hline Once a month & $94 / 228$ & 41.2 & $4.615(2.998-7.104)$ & $3.411(1.746-6.665)$ \\
\hline Once a week & $47 / 92$ & 51.1 & $6.871(4.034-11.703)$ & $2.647(1.030-6.803)$ \\
\hline \multicolumn{5}{|c|}{ Reported consumption of unrecorded alcohol } \\
\hline Yes & $127 / 430$ & 29.5 & $1.166(0.851-1.599)$ & $0.897(0.596-1.349)$ \\
\hline No & & & 1.0 & 1.0 \\
\hline \multicolumn{5}{|c|}{ Reported encountering COVID-19-related restrictions in public life in the past 3 months } \\
\hline No restrictions & $12 / 62$ & 19.4 & 1.0 & 1.0 \\
\hline Slight restrictions & $48 / 266$ & 18.0 & $0.917(0.454-1.854)$ & $0.943(0.263-3.379)$ \\
\hline Moderate restrictions & $79 / 239$ & 33.1 & $2.057(1.037-4.082)$ & $1.553(0.381-6.329)$ \\
\hline Severe restrictions & $81 / 224$ & 36.2 & $2.360(1.188-4.689)$ & $0.820(0.187-3.599)$ \\
\hline \multicolumn{5}{|c|}{ Reported encountering COVID-19-related restrictions in their everyday private life in the past 3 months } \\
\hline No restrictions & $12 / 83$ & 14.5 & 1.0 & 1.0 \\
\hline Slight restrictions & $57 / 295$ & 19.3 & $1.417(0.720-2.787)$ & $1.513(0.467-4.899)$ \\
\hline Moderate restrictions & $78 / 222$ & 35.1 & $3.205(1.638-6.269)$ & $2.690(0.720-10.055)$ \\
\hline Severe restrictions & $75 / 191$ & 39.3 & $3.825(1.944-7.530)$ & $3.889(0.969-15.608)$ \\
\hline \multicolumn{5}{|c|}{ Reported negative consequences of the pandemic to their professional or financial situation in the past 3 months } \\
\hline No negative consequences & 40/195 & 20.5 & 1.0 & 1.0 \\
\hline Slight negative consequences & $72 / 306$ & 23.5 & $1.192(0.771-1.845)$ & $0.699(0.383-1.275)$ \\
\hline Moderate negative consequences & $54 / 153$ & 35.3 & $2.114(1.307-3.417)$ & $1.280(0.625-2.621)$ \\
\hline Severe negative consequences & $57 / 132$ & 43.2 & $2.945(1.805-4.804)$ & $2.329(1.001-5.428)$ \\
\hline \multicolumn{5}{|c|}{ Reported confirmed SARS-CoV-2 infection in themselves, their family or friends in the past 3 months) } \\
\hline Yes & $48 / 171$ & 28.1 & $0.986(0.676-1.438)$ & $0.893(0.554-1.439)$ \\
\hline No & $171 / 603$ & 28.4 & 1.0 & 1.0 \\
\hline \multicolumn{5}{|c|}{ Reported stress due to the spread of SARS-CoV-2 } \\
\hline Yes & $140 / 423$ & 33.1 & 1.749 (1.251-2.466) & $1.004(0.628-1.606)$ \\
\hline No & $69 / 313$ & 22.0 & 1.0 & 1.0 \\
\hline
\end{tabular}

Note: * - respondents who had never drunk 6 or more alcoholic drinks on a single occasion in the past 12 months were excluded from the analysis.

\section{Factors associated with the increase in the frequency of heavy episodic drinking during the first months of the pandemic}

Positive statistically significant associations were identified between the increase in the frequency of heavy episodic drinking during the pandemic and the following factors: drinking alcohol 2-3 times a week (OR: 2.756; 95\% Cl: 1.137-6.679) or more often before the pandemic (OR: $6.581 ; 95 \% \mathrm{Cl}: 2.585-$
16.749), having 7 or more drinks on a typical drinking occasion before the pandemic (OR: 2.202; 95\% Cl: 1.014-4.779) and heavy episodic drinking once a month or more often before the pandemic (from OR: 2.561; 95\% Cl: $1.341-4.893$ to OR: 2.647; 95\% Cl: 1.030-6.803) (Table 4). The odds of increase in the frequency of heavy episodic drinking were significantly more than two times higher among persons who reported severe negative consequences of the pandemic to their financial or professional situation (OR: 2.329; 95\% Cl: 1.001-5.428). 


\section{DISCUSSION}

The associations revealed in our study are corroborated by other research works conducted in various countries during the first months of the pandemic. Specifically, in an Israeli study male sex was associated with increased consumption of beer and strong liquors [16], whereas in another study conducted in Canada male sex, stress, the feelings of isolation and hopelessness were associated with increased frequency of alcohol use in the first months of the pandemic [17]. In a UK study, stress caused by the pandemic was associated with the rise in hazardous drinking [18]. High levels of anxiety and stress caused by the pandemic led to the increase in alcohol use in Australia, France and Canada [17, 19, 20].

A few studies investigated changes in alcohol consumption in the general population unstratified by pandemic-related factors. Thus, a French publication reported an increase in total alcohol consumption during the lockdown [20]; by contrast, alcohol use in Greece and Spain during the lockdown was declining [21, 22]. However, in Greece and Spain the decline was less pronounced among stressed individuals and those with low or middle income. Compared to the prepandemic level, alcohol was purchased in larger quantities during the lockdown by Russian [23, 24] and UK households [25]. During the first months of the pandemic, retail alcohol sales were on the rise in the US [26]. A global study investigating changes in the availability and use of psychoactive drugs and alcohol during the pandemic reported a $71 \%$ increase in alcohol consumption across the world [27]. However, according to another large-scale study, alcohol consumption in the general population decreased in most European countries, including Russia, during the first months of the pandemic, mostly due to the reduction in the frequency of heavy episodic drinking [22].

Our study discovered a significant increase in 3 key parameters of alcohol consumption during the first months of the pandemic among those individuals who initially had consumed alcohol in larger quantities and more frequently. These key parameters include the frequency of drinking, the usual consumption (volume) of alcohol on a typical drinking occasion and the frequency of heavy episodic drinking. These findings suggest polarization of alcohol use, i.e. a situation when alcohol consumption grows among those who drink more at baseline and declines among those who typically drink less. This is consistent with the results of another online survey conducted in the general adult population of Russia [28]. The associations established in our study between the increase in the frequency of alcohol use/ heavy episodic drinking and such COVID-19-related factors as severe restrictions of everyday private life and the negative consequences of the pandemic to professional or financial situation uncover a new aspect or mechanism associated with stress which drives changes in drinking behaviors during the pandemic. Our findings go in line with the stress-associated patterns reported by the studies mentioned above and are consistent with previously reported changes in drinking behavior among Russians determined by certain sociodemographic characteristics, environmental factors and stress in times of socioeconomic and public health crises [29].

A potential limitation of this study might be the insufficient representativeness of the surveyed sample of online social media users in Russia. However, the size of this selection bias is likely small, because the survey was conducted among the users of social networking services highly popular in Russia and because the sociodemographic characteristics of the respondents were generally the same as those of the vast majority of online social media users in Russia. Besides, the consistency of the established patterns and associations with the results of other studies suggests that the probability of our results not being the consequence of selection bias is high. Another limitation of this study is that changes in alcohol consumption were assessed without differentiating between recorded and unrecorded alcohol. Therefore, additional analysis is needed to investigate changes in the consumption of unrecorded alcohol [30], considering its fairly high availability in Russia during the COVID-19 pandemic [31].

\section{CONCLUSIONS}

During the first months of the COVID-19 pandemic, over onethird of male and quarter of female online social media users in Russia increased the frequency of drinking. One in four men and one in five women increased their usual consumption (volume) of alcohol on a typical drinking occasion, and about one-third of men and women engaged in heavy episodic drinking more frequently than before the pandemic. Increased alcohol consumption during the first months of the pandemic was associated with male sex, younger age, severe restrictions imposed on everyday private life and severe negative consequences of the pandemic to the professional or financial situation. Further research is needed to investigate the possibility of implementing screening and brief interventions via online social media to prevent problems associated with alcohol use during large-scale epidemics and public health crises.

\section{References}

1. RosStat (Federal'naja sluzhba gosudarstvennoj statistiki). [procitirovano 18.10.2021]. Official Statistics. Dostupno po ssylke: https://eng.gks.ru/folder/11335.

2. Artamonov RE, Lavrentev NV. Opros obshhestvennogo mnenija naselenija RF. Izmenenija, vyzvannye jepidemiej koronavirusa i rezhimom samoizoljacii. Analiticheskij bjulleten' NIU VShJe. 2020; (3): 4-24. Available from: https://www.hse.ru/mirror/pubs/ share/368507661.pdf.

3. McPhee MD, Keough MT, Rundle S, Heath LM, Wardell JD, Hendershot CS. Depression, Environmental Reward, Coping Motives and Alcohol Consumption During the COVID-19 Pandemic. Front Psychiatry. 2020; 11: 574676. PMID: 33192708; PMCID: PMC7661794. Available from: https://doi.org/10.3389/ fpsyt.2020.574676.

4. Hamza CA, Ewing L, Heath NL, Goldstein AL. When social isolation is nothing new: a longitudinal study on psychological distress during COVID-19 among university students with and without preexisting mental health concerns. Can Psychol.

2021; 62 (1): 20-30. Available from: https://doi.org/10.1037/ cap0000255.

5. Kuntsche E, Gabhainn SN, Roberts C, Windlin B, Vieno A, Bendtsen $P$, et al. Drinking motives and links to alcohol use in 13 European countries. J Stud Alcohol Drugs. 2014; 75 (3): 428-37. PMID: 24766755. Available from: https://10.15288/ jsad.2014.75.428.

6. Cooper ML, Kuntshce E, Levitt A, Barber LL, Wolf S. Motivational models of substance use: a review of theory and research on motives for using alcohol, marijuana, and tobacco. In: Sher KJ, editor. The Oxford handbook of substance use and substance use disorders. 1st edition. Oxford (UK): Oxford University Press, 2016; p. 375-421.

7. Brooks SK, Webster RK, Smith LE, Woodland L, Wessely S, Greenberg N, et al. The psychological impact of quarantine and how to reduce it: rapid review of the evidence. Lancet. 2020; 395 (10227): 912-20. PMID: 32112714; PMCID: PMC7158942. Available from: https://10.1016/S0140-6736(20)30460-8. 
8. de Goeij MC, Suhrcke M, Toffolutti V, van de Mheen D, Schoenmakers TM, Kunst AE. How economic crises affect alcohol consumption and alcohol-related health problems: a realist systematic review. Soc Sci Med. 2015; 131: 131-46. PMID: 25771482. Available from: https://10.1016/.j.socscimed.2015.02.025

9. Alexander AC, Ward KD. Understanding Postdisaster Substance Use and Psychological Distress Using Concepts from the Self-Medication Hypothesis and Social Cognitive Theory. J Psychoactive Drugs. 2018; 50 (2): 177-186. PMID: 29125424; PMCID: PMC6101235. Available from: htt ps://10.1080/02791072.2017.1397304

10. Rehm J, Kilian C, Ferreira-Borges C, Jernigan D, Monteiro $\mathrm{M}$, Parry $\mathrm{CDH}$, et al. Alcohol use in times of the COVID 19: Implications for monitoring and policy. Drug Alcohol Rev. 2020; 39 (4): 301-4. PMID: 32358884; PMCID: PMC7267161. Available from: https://10.1111/dar.13074.

11. Jacob L, Smith L, Armstrong NC, Yakkundi A, Barnett $Y$, Butler L, et al. Alcohol use and mental health during COVID-19 lockdown: A cross-sectional study in a sample of UK adults. Drug Alcohol Depend. 2021; 219: 108488. PMID: 33383352; PMCID: PMC7768217. Available from: https://10.1016/j.drugalcdep.2020.108488.

12. Rodriguez LM, Litt DM, Stewart SH. Drinking to cope with the pandemic: The unique associations of COVID-19-related perceived threat and psychological distress to drinking behaviors in American men and women. Addict Behav. 2020; 110: 106532. PMID: 32652385; PMCID: PMC7320671. Available from: https:// doi.org/10.1016/j.addbeh.2020.106532.

13. Rotermann M. Canadians who report lower self-perceived mental health during the COVID-19 pandemic more likely to report increased use of cannabis, alcohol and tobacco. Ottawa (ON): Statistics Canada; 2020 [Statistics Canada, Catalogue No.: 45280001]. [cited 19.10.2021]. Available from: https:// www150.statcan.gc.ca/n1/en/pub/45-28-0001/2020001/ article/00008-eng. pdf?st=B500340M.

14. Bambra C, Riordan R, Ford J, Matthews F. The COVID-19 pandemic and health inequalities. J Epidemiol Community Health. 2020; 74 (11): 964-8. Available from: https://doi.org/10.1136/jech-2020-214401.

15. Vigo D, Patten S, Pajer K, Krausz M, Taylor S, Rush B, et al. Mental health of communities during the COVID-19 pandemic. Can J Psychiatry. 2020; 65 (10): 681-7. Available from: https:// doi.org/10.1177/0706743720926676

16. Levy I, Cohen-Louck K, Bonny-Noach H. Gender, employment, and continuous pandemic as predictors of alcohol and drug consumption during the COVID-19. Drug Alcohol Depend. 2021; 228: 109029. PMID: 34500243; PMCID: PMC8411591. Available from: https://10.1016/j.drugalcdep.2021.109029.

17. Thompson K, Dutton DJ, MacNabb K, Liu T, Blades S, Asbridge M. Changes in alcohol consumption during the COVID-19 pandemic: exploring gender differences and the role of emotional distress. Health Promot Chronic Dis Prev Can. 2021; 41 (9): 254-263. English, French. PMID: 34164972. Available from: https://10.24095/hpcdp.41.9.02.

18. Clay JM, Stafford LD, Parker MO. Associations Between Selfreported Inhibitory Control, Stress, and Alcohol (Mis)use During the First Wave of the COVID-19 Pandemic in the UK: a National Crosssectional Study Utilising Data From Four Birth Cohorts. Int J Ment Health Addict. 2021: 1-22. PMID: 34366730; PMCID: PMC8330475. Available from: https://10.1007/s11469-021-00599-8.

19. Rolland B, Haesebaert F, Zante E, Benyamina A, Haesebaert J, Franck N. Global Changes and Factors of Increase in Caloric/
Salty Food Intake, Screen Use, and Substance Use During the Early COVID-19 Containment Phase in the General Population in France: Survey Study. JMIR Public Health Surveill. 2020; 6 (3): e19630. Available from: https://doi.org/10.2196/19630. Erratum in: JMIR Public Health Surveill. 2021; 7 (7): e31906. PMID: 32589149; PMCID: PMC7505683.

20. Stanton R, To QG, Khalesi S, Williams SL, Alley SJ, Thwaite TL, et al. Depression, Anxiety and Stress during COVID-19: Associations with Changes in Physical Activity, Sleep, Tobacco and Alcohol Use in Australian Adults. Int J Environ Res Public Health. 2020; 17 (11): 4065. PMID: 32517294; PMCID: PMC7312903. Available from: https://10.3390/ijerph17114065.

21. Panagiotidis P, Rantis K, Holeva V, Parlapani E, Diakogiannis I. Changes in Alcohol Use Habits in the General Population, during the COVID-19 Lockdown in Greece. Alcohol Alcohol. 2020; 55 (6): 702-4. PMID: 32959877; PMCID: PMC7543262. Available from: https://doi. org/10.1093/alcalc/agaa092.

22. Kilian C, Rehm J, Allebeck P, Braddick F, Gual A, Barták M, et al. Alcohol consumption during the COVID-19 pandemic in Europe: a large-scale cross-sectional study in 21 countries. Addiction. 2021. PMID: 34109685. Available from: https://10.1111/add.15530.

23. Issledovanija na osnove dannyh Sberbanka. 2020. [procitirovano 15.10.2021]. Dostupno po ssylke: https://www.sberbank.ru/ru/ about/issledovaniya

24. Nemcov AV, Gridin RR. Kosvennye pokazateli potreblenija alkogolja vo vremja jepidemii koronavirusa v Rossii. Voprosy narkologii. 2020; (10): 16-33. Available from: htt ps://10.47877/0234-0623_2020_10_16.

25. Anderson P, Llopis EJ, O'Donnell A, Kaner E. Impact of COVID-19 Confinement on Alcohol Purchases in Great Britain: Controlled Interrupted Time-Series Analysis During the First Half of 2020 Compared With 2015-2018. Alcohol Alcohol. 2021; 56 (3): 30716. PMID: 33211796; PMCID: PMC7717153. Available from: https://doi.org/10.1093/alcalc/agaa128.

26. Castaldelli-Maia JM, Segura LE, Martins SS. The concerning increasing trend of alcohol beverage sales in the U.S. during the COVID-19 pandemic. Alcohol. 2021; 96: 37-42. PMID: 34245809; PMCID: PMC8421038. Available from: https://10.1016/j.alcohol.2021.06.004.

27. Farhoudian A, Radfar SR, Mohaddes Ardabili H, Rafei P, Ebrahim M, et al. A Global Survey on Changes in the Supply, Price, and Use of Illicit Drugs and Alcohol, and Related Complications During the 2020 COVID-19 Pandemic. Front Psychiatry. 2021; 12: 646206. PMID: 34421664; PMCID: PMC8377291. Available from: https://10.3389/fpsyt.2021.646206.

28. Gil AYu, Vyshinskiy KV, Fadeeva EV, Khalfin R. A. Izmenenija osobennostej potreblenija alkogolja $v$ Rossijskoj Federacii $v$ pervye mesjacy pandemii COVID-19. Problemy standartizacii v zdravoohranenii. 2021; 5-6: 63-73. Available from: https://doi. org/10.26347/1607-2502202105-06063-073.

29. Nemtsov AV. A Contemporary History of Alcohol in Russia. Södertörn: Södertörn University, 2011; 348 r.

30. Gil A, Khalfin R, Krinitsky S, Nikoforov S, Ilchenko I, Kosagovskaya I, i dr. Upotreblenie surrogatnogo alkogolja licami s rasstrojstvami, svjazannymi so zloupotrebleniem alkogolem (na primere sub"ekta Rossijskoj Federacii). Problemy social'noj gigieny, zdravoohranenija i istorii mediciny. 2021; 29 (3): 486-91. Available from: https://doi. org/10.32687/0869-866X-2021-29-3-486-491.

31. Gil A. COVID-19: A need for stricter control over unrecorded alcohol in Russia. Adicciones. 2021; 33 (3): 281-4. Available from: https://doi.org/10.20882/adicciones.1634

\section{Литература}

1. РосСтат (Федеральная служба государственной статистики). [процитировано 18.10.2021]. Official Statistics. Доступно по ссылке: https://eng.gks.ru/folder/11335.

2. Артамонов Р. Е., Лаврентьев Н. В. Опрос общественного мнения населения РФ. Изменения, вызванные эпидемией коронавируса и режимом самоизоляции. Аналитический бюллетень НИУ ВШЭ. 2020; (3): 4-24. Доступно по ссылке: https://www.hse.ru/mirror/pubs/share/368507661.pdf

3. McPhee MD, Keough MT, Rundle S, Heath LM, Wardell JD, Hendershot CS. Depression, Environmental Reward, Coping

Motives and Alcohol Consumption During the COVID-19 Pandemic. Front Psychiatry. 2020; 11: 574676. PMID: 33192708; PMCID: PMC7661794. Available from: https://doi.org/10.3389/ fpsyt.2020.574676.

4. Hamza CA, Ewing L, Heath NL, Goldstein AL. When social isolation is nothing new: a longitudinal study on psychological distress during COVID-19 among university students with and without preexisting mental health concerns. Can Psychol. 2021; 62 (1): 20-30. Available from: https://doi.org/10.1037/ cap0000255 
5. Kuntsche E, Gabhainn SN, Roberts C, Windlin B, Vieno A, Bendtsen $\mathrm{P}$, et al. Drinking motives and links to alcohol use in 13 European countries. J Stud Alcohol Drugs. 2014; 75 (3): 428-37. PMID: 24766755. Available from: https://10.15288/ jsad.2014.75.428.

6. Cooper ML, Kuntshce E, Levitt A, Barber LL, Wolf S. Motivational models of substance use: a review of theory and research on motives for using alcohol, marijuana, and tobacco. In: Sher KJ, editor. The Oxford handbook of substance use and substance use disorders. 1st edition. Oxford (UK): Oxford University Press, 2016; p. 375-421.

7. Brooks SK, Webster RK, Smith LE, Woodland L, Wessely S, Greenberg N, et al. The psychological impact of quarantine and how to reduce it: rapid review of the evidence. Lancet. 2020; 395 (10227): 912-20. PMID: 32112714; PMCID: PMC7158942. Available from: https://10.1016/S0140-6736(20)30460-8.

8. de Goeij MC, Suhrcke M, Toffolutti V, van de Mheen D, Schoenmakers TM, Kunst AE. How economic crises affect alcohol consumption and alcohol-related health problems: a realist systematic review. Soc Sci Med. 2015; 131: 13146. PMID: 25771482. Available from: https://10.1016/j. socscimed.2015.02.025

9. Alexander AC, Ward KD. Understanding Postdisaster Substance Use and Psychological Distress Using Concepts from the Self-Medication Hypothesis and Social Cognitive Theory. J Psychoactive Drugs. 2018; 50 (2): 177-186. PMID: 29125424; PMCID: PMC6101235. Available from: htt ps://10.1080/02791072.2017.1397304.

10. Rehm J, Kilian C, Ferreira-Borges C, Jernigan D, Monteiro $\mathrm{M}$, Parry $\mathrm{CDH}$, et al. Alcohol use in times of the COVID 19: Implications for monitoring and policy. Drug Alcohol Rev. 2020; 39 (4): 301-4. PMID: 32358884; PMCID: PMC7267161. Available from: https://10.1111/dar.13074.

11. Jacob L, Smith L, Armstrong NC, Yakkundi A, Barnett $Y$, Butler L, et al. Alcohol use and mental health during COVID-19 lockdown: A cross-sectional study in a sample of UK adults. Drug Alcohol Depend. 2021; 219: 108488. PMID: 33383352; PMCID: PMC7768217. Available from: https://10.1016/j. drugalcdep.2020.108488.

12. Rodriguez LM, Litt DM, Stewart SH. Drinking to cope with the pandemic: The unique associations of COVID-19-related perceived threat and psychological distress to drinking behaviors in American men and women. Addict Behav. 2020; 110: 106532. PMID: 32652385; PMCID: PMC7320671. Available from: https:// doi.org/10.1016/j.addbeh.2020.106532.

13. Rotermann M. Canadians who report lower self-perceived mental health during the COVID-19 pandemic more likely to report increased use of cannabis, alcohol and tobacco. Ottawa (ON): Statistics Canada; 2020 [Statistics Canada, Catalogue No.: 45280001]. [cited 19.10.2021]. Available from: https:// www150.statcan.gc.ca/n1/en/pub/45-28-0001/2020001/ article/00008-eng.pdf?st=B5०O340M.

14. Bambra C, Riordan R, Ford J, Matthews F. The COVID-19 pandemic and health inequalities. J Epidemiol Community Health. 2020; 74 (11): 964-8. Available from: https://doi.org/10.1136/ jech-2020-214401.

15. Vigo D, Patten S, Pajer K, Krausz M, Taylor S, Rush B, et al. Mental health of communities during the COVID-19 pandemic. Can J Psychiatry. 2020; 65 (10): 681-7. https://doi. org/10.1177/0706743720926676.

16. Levy I, Cohen-Louck K, Bonny-Noach H. Gender, employment, and continuous pandemic as predictors of alcohol and drug consumption during the COVID-19. Drug Alcohol Depend. 2021; 228: 109029. PMID: 34500243; PMCID: PMC8411591. Available from: https://10.1016/j.drugalcdep.2021.109029.

17. Thompson K, Dutton DJ, MacNabb K, Liu T, Blades S, Asbridge M. Changes in alcohol consumption during the COVID-19 pandemic: exploring gender differences and the role of emotional distress. Health Promot Chronic Dis Prev Can. 2021; 41 (9): 254-263. English, French. PMID: 34164972. Available from: https://10.24095/hpcdp.41.9.02.

18. Clay JM, Stafford LD, Parker MO. Associations Between Selfreported Inhibitory Control, Stress, and Alcohol (Mis)use During the First Wave of the COVID-19 Pandemic in the UK: a National Cross-sectional Study Utilising Data From Four Birth Cohorts. Int J Ment Health Addict. 2021: 1-22. PMID: 34366730; PMCID: PMC8330475. Available from: https://10.1007/s11469-02100599-8.

19. Rolland B, Haesebaert F, Zante E, Benyamina A, Haesebaert J, Franck N. Global Changes and Factors of Increase in Caloric/ Salty Food Intake, Screen Use, and Substance Use During the Early COVID-19 Containment Phase in the General Population in France: Survey Study. JMIR Public Health Surveill. 2020; 6 (3): e19630. Available from: https://doi.org/10.2196/19630. Erratum in: JMIR Public Health Surveill. 2021; 7 (7): e31906. PMID: 32589149; PMCID: PMC7505683.

20. Stanton R, To QG, Khalesi S, Williams SL, Alley SJ, Thwaite TL, et al. Depression, Anxiety and Stress during COVID-19: Associations with Changes in Physical Activity, Sleep, Tobacco and Alcohol Use in Australian Adults. Int J Environ Res Public Health. 2020; 17 (11): 4065. PMID: 32517294; PMCID: PMC7312903. Available from: https://10.3390/ijerph17114065.

21. Panagiotidis $P$, Rantis K, Holeva V, Parlapani E, Diakogiannis I. Changes in Alcohol Use Habits in the General Population, during the COVID-19 Lockdown in Greece. Alcohol Alcohol. 2020; 55 (6): 702-4. PMID: 32959877; PMCID: PMC7543262. Available from: https://doi.org/10.1093/alcalc/ agaa092.

22. Kilian C, Rehm J, Allebeck P, Braddick F, Gual A, Barták M, et al. Alcohol consumption during the COVID-19 pandemic in Europe: a large-scale cross-sectional study in 21 countries. Addiction. 2021. PMID: 34109685. Available from: https://10.1111/add.15530.

23. Исследования на основе данных Сбербанка. 2020. [процитировано 15.10.2021]. Доступно по ссылке: https:// www.sberbank.ru/ru/about/issledovaniya

24. Немцов А. В., Гридин Р. Р. Косвенные показатели потребления алкоголя во время эпидемии коронавируса в России. Вопросы наркологии. 2020; (10): 16-33. Доступно по ссылке: https://10.47877/0234-0623_2020_10_16.

25. Anderson P, Llopis EJ, O'Donnell A, Kaner E. Impact of COVID-19 Confinement on Alcohol Purchases in Great Britain: Controlled Interrupted Time-Series Analysis During the First Half of 2020 Compared With 2015-2018. Alcohol Alcohol. 2021; 56 (3): 30716. PMID: 33211796; PMCID: PMC7717153. Available from: https://doi.org/10.1093/alcalc/agaa128.

26. Castaldelli-Maia JM, Segura LE, Martins SS. The concerning increasing trend of alcohol beverage sales in the U.S. during the COVID-19 pandemic. Alcohol. 2021; 96: 37-42. PMID: 34245809; PMCID: PMC8421038. Available from: https://10.1016/j. alcohol.2021.06.004.

27. Farhoudian A, Radfar SR, Mohaddes Ardabili H, Rafei P, Ebrahimi M, et al. A Global Survey on Changes in the Supply, Price, and Use of Illicit Drugs and Alcohol, and Related Complications During the 2020 COVID-19 Pandemic. Front Psychiatry. 2021; 12: 646206. PMID: 34421664; PMCID: PMC8377291. Available from: https://10.3389/fpsyt.2021.646206.

28. Гиль А. Ю., Вышинский К. В., Фадеева Е. В., Хальфин Р. А. Изменения особенностей потребления алкоголя в Российской Федерации в первые месяцы пандемии COVID-19. Проблемы стандартизации в здравоохранении. 2021; 5-6: 63-73. Доступно по ссылке: https://doi. org/10.26347/1607-2502202105-06063-073.

29. Nemtsov AV. A Contemporary History of Alcohol in Russia. Södertörn: Södertörn University, 2011; 348 p.

30. Гиль А. Ю., Хальфин Р. А., Криницкий С. М., Никифоров С. А., Ильченко И. Н., Косаговская И. И., и др. Употребление суррогатного алкоголя лицами с расстройствами, связанными со злоупотреблением алкоголем (на примере субъекта Российской Федерации). Проблемы социальной гигиены, здравоохранения и истории медицины. 2021; 29 (3): 486-91. Доступно по ссылке: https://doi.org/10.32687/0869866X-2021-29-3-486-491.

31. Gil A. COVID-19: A need for stricter control over unrecorded alcohol in Russia. Adicciones. 2021; 33 (3): 281-4. Available from: https://doi.org/10.20882/adicciones.1634. 